\title{
Successful use of percutaneous ECMO and intra-aortic balloon support for a rescue valve in valve TAVR procedure in a patient in cardiogenic shock
}

\author{
Alenka Golicnik ${ }^{1 *}$, Matjaz Bunc ${ }^{2}$, Marko Noc ${ }^{1}$ and Vojka Gorjup ${ }^{1}$ \\ ${ }^{1}$ Department for Intensive Internal Medicine, University Medical Center Ljubljana, Slovenia \\ ${ }^{2}$ Department for Cardiology, University Medical Center Ljubljana, Slovenia
}

\begin{abstract}
Transcatheter aortic valve replacement (TAVR) is becoming an effective treatment method for severe aortic stenosis but can pose severe hemodynamic risk during procedure. Acknowledgement of the risk and a plan for preprocedural hemodynamic stabilization of high-risk patients using advanced hemodynamic support measures is therefore crucial for final success. We report and discuss a case of a patient in cardiogenic shock due to left ventricular failure because of a severe redo biological AVR stenosis that was successfully treated with a rescue valve in valve TAVR under prophylactic pre- and postprocedural percutaneous mechanical support with a combination of VA ECMO and intra-aortic balloon pump (IABP).
\end{abstract}

\section{Introduction}

Transcatheter aortic valve replacement (TAVR) is an effective method for treating severe aortic stenosis in high risk surgical patients that is recently being expanded also to intermediate-risk populations [1-3]. TAVR has also been used as a treatment for the failing bio prostheses as a valve in valve procedure with mortality rates similar to those in redo surgical AVR despite higher surgical risk [4]. However, TAVR procedure can cause profound hemodynamic destabilization in patients at an already high risk of complications. It is therefore crucial that patients with severe left ventricular dysfunction, acute heart failure or cardiogenic shock are hemodynamically stabilized peri procedurally. Veno-arterial (VA) ECMO offers an option of mechanical circulatory support. There is still little evidence on the preprocedural prophylactic use of VA ECMO in critically ill TAVR patients [5]. On the other hand, data is emerging on increased VA ECMO weaning success and increased survival when ECMO is used in combination with intra-aortic balloon pump (IABP) in patients with cardiogenic shock [6]. We report a case of a patient in cardiogenic shock due to left ventricular failure because of a severe redo biological AVR stenosis that was successfully treated with a rescue valve in valve TAVR under prophylactic pre- and postprocedural percutaneous mechanical support with a combination of VA ECMO and IABP.

\section{Case Report}

A 56 years old male was admitted to cardiology department due to symptoms of cardiac decompensation that presented as breathlessness on minimal exertion, dizziness and orthopnea. He was in NYHA class III. Past medical history revealed that he had undergone a mechanical AVR in 1992 due to congenital aortic stenosis. In 2014 a mechanical re-AVR was performed because of valve degeneration and an aortic graft was implanted at the same time due to ascending aorta aneurysm. In 2015 he was diagnosed with AVR endocarditis and was reoperated with a biological AVR and aortic graft implantation. Two years later severe aortic regurgitation was observed, and he was reoperated for the fourth time and a new biological AVR (Sorin Perceval L $25 \mathrm{~mm}$ ) was implanted.

During this hospitalization echocardiography revealed a severe stenosis of biological AVR due to pannus formation (mean gradient $44 \mathrm{~mm} \mathrm{Hg}$, AVA $0.4 \mathrm{~cm}^{2}$ ), a dilated left ventricle (EDD $6 \mathrm{~cm}$ ) with minimal concentric hypertrophy, a severely reduced left ventricular ejection fraction of $20 \%$ (SVi $27 \mathrm{ml} / \mathrm{m}^{2}$ ) and a moderate postcapillar pulmonary hypertension (sPAP $50 \mathrm{~mm} \mathrm{Hg}$ ). Coronary angiogram was normal (Figures 1 and 2). His surgical risk stratification at that time revealed a Euroscore II of $10.98 \%$. After heart team discussion he was planned for another mechanical re-AVR (Bentall procedure). Due to a higher surgical risk, reduced ejection fraction and young age a protocol for elective heart transplantation listing was started as well. During the course of hospitalization diuretic therapy was intensified and he received levosimendan infusion. In spite of this his condition deteriorated and on day 20 he had a syncope. Sustained VT was observed that resolved spontaneously but patient developed pulmonary edema and cardiogenic shock. He was transferred to ICU where he was intubated and mechanically ventilated, IABP was inserted and he was put on high inotropic and vasopressor support to maintain arterial pressure and organ perfusion. In spite of all these measures patient's condition deteriorated further, he was hypotensive with a MAP $60 \mathrm{mmHg}$, a short

*Correspondence to: Alenka Golicnik, Department for Intensive Internal Medicine, University Medical Center Ljubljana, Zaloska 7, 1000 Ljubljana, Slovenia, E-mail: alenka.golicnik@kclj.si

Key words: transcatheter aortic valve replacement (TAVR), cardiogenic shock, extracorporeal membrane oxygenation (ECMO), intra-aortic balloon pump $(I A B P)$

Received: February 23, 2019; Accepted: March 19, 2019; Published: March 22, 2019 

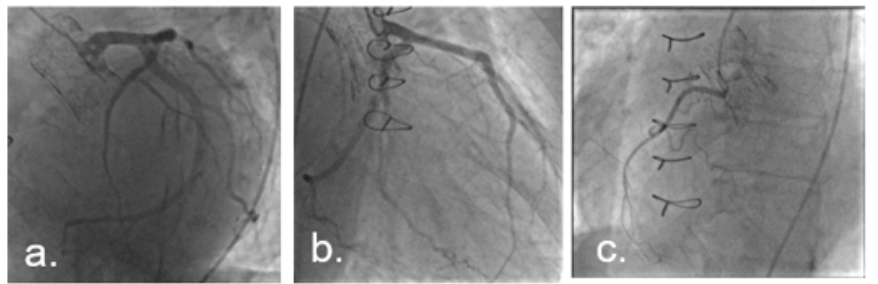

Figure 1. Coronary angiogram before TAVR. No important coronary lesions. a. LAO CAU (LCA) b. RAO (LCA) c. LAO (RCA)
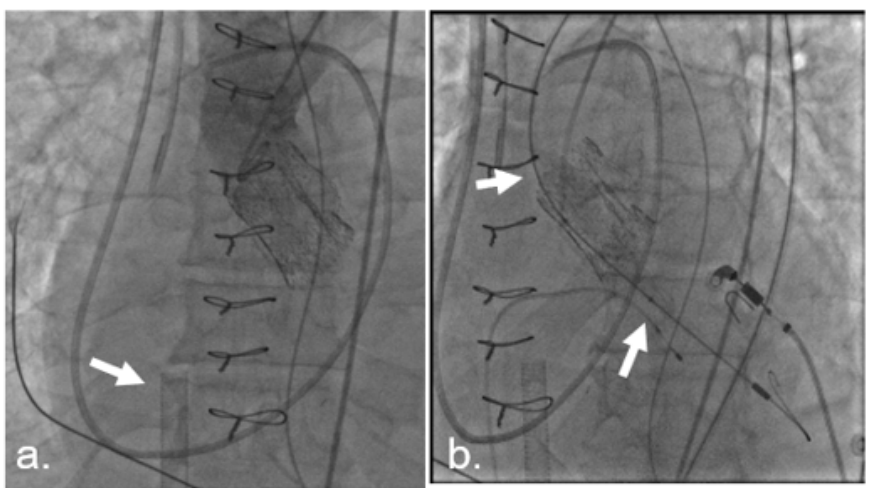

Figure 2. Aortic angiography before TAVR. No aortic regurgitation, degenerated Perceval (Sorin, USA) valve. a. aortic angiography: degenerated Perceval (Sorin) valve. ECMO cannula (arrow) b. Baloon aortic valvuloplasty

resuscitation was needed due to PEA, his kidney and liver function deteriorated, and anuria developed. Swan-Ganz catheterization revealed high right and left filling pressures (CVP $27 \mathrm{mmHg}$, sPAP 63 $\mathrm{mmHg}$, dPAP $40 \mathrm{mmHg}$, PWP $40 \mathrm{mmHg}$ ) with a low CI of $1.9 \mathrm{~L} / \mathrm{min} /$ $\mathrm{m}^{2}$. As we could not stabilize the patient further treatment options were discussed with heart team and a decision was made to try to achieve patient stabilization with a rescue valve in valve TAVR procedure done under a percutaneously implanted ECMO support in view of high-risk unstable patient in cardiogenic shock.

After transfer to catheterization laboratory right femoral artery and vein were cannulated first with a $15 \mathrm{Fr}$ arterial and $23 \mathrm{Fr}$ venous cannula and patient was connected to ECMO circuit (Cardiohelp, Maquet) [7]. Flow rate was set to $1 \mathrm{lpm}$. IABP previously placed trough a 7 Fr sheath in left femoral artery was removed and an 18 Fr sheath was placed for the TAVI prosthesis delivery. Balloon aortic valvuloplasty was done with Zelos baloon (OptiMed, UK) $20 \times 40 \mathrm{~mm}$ during $180 / \mathrm{min}$ rapid ventricular pacing to predilate the valve. Post predilation there was a loss of arterial pressure pulsatility observed with a MAP of $30 \mathrm{mmHg}$ as there was no significant cardiac output post procedure and ECMO flow was increased to $4 \mathrm{lpm}$ during that time to preserve organ and coronary perfusion. Edwards Sapien 326 $\mathrm{mm}$ valve was then positioned and deployed. There was an immediate arterial pulsatility restoration with a left vetricular systolic pressure of $110 \mathrm{mmHg}$ after placement of the valve. ECMO flow was reduced slowly back to $1 \mathrm{lpm}$ with preservation of arterial pressure pulsatility and MAP. There was no significant aortic regurgitation observed post procedure. TAVI sheath was removed and percutaneous approach was closed with a combination of Proglide and Angioseal closure systems. IABP was inserted again trough left femoral artery and patient was transferred back to ICU on low flow VA ECMO and IABP support.

Immediately after TAVR procedure we observed patient stabilization with massive diuresis ensuing and an increase in CI to
$3.7 \mathrm{l} / \mathrm{min} / \mathrm{m} 2$ with a reduction of filling pressures $(\mathrm{CVP} 7 \mathrm{mmHg}$, sPAP $35 \mathrm{mmHg}$, dPAP $22 \mathrm{mmHg}$, PWP $13 \mathrm{mmHg}$ ). After another 4 hours of stable hemodynamics on low ECMO flow of $1 \mathrm{lpm}$, ECMO circuit was removed. IABP was removed on the next day. There were no complications on percutaneous approach sites. Echocardiography post procedurally revealed an improvement in LVEF to 30\% with a good functioning of positioned aortic valve with a mean transprothetic gradient of $17 \mathrm{mmHg}$, AVA $2.5 \mathrm{~cm} 2$, and mild pulmonary hypertension (sPAP $28 \mathrm{~mm} \mathrm{Hg}$ ). He also received levosimendan. Heart failure reversed rapidly, we observed improvement of kidney and liver function. Pleural drainage was performed to improve patient recompensation and on day 5 the patient was successfully extubated and then transferred back to cardiology department after 6 days of ICU stay.

During further hospital stay patient completed protocol for inclusion into heart transplantation list. CRT-D was also implanted, and he was rehabilitated and released into home care with a normal kidney function and in NYHA II class. He is being followed-up regularly and is listed into transplantation waiting list. His latest echocardiography showed a dilated left ventricle $($ EDD $5.8 \mathrm{~cm})$ with moderately decreased ejection fraction (LVEF 46\%), good functioning of prosthetic valve (mean transprothetic gradient $20 \mathrm{mmHg}$, AVA $2.01 \mathrm{~cm}^{2}$, minimal paravalvular leak) and mild pulmonary hypertension (sPAP $29 \mathrm{mmHg}$ ) (Figure. 3).

\section{Discussion}

TAVR seems an attractive treatment option for high risk redo patients as conventional elective redo aortic valve surgery carries an operative mortality risk from $2-7 \%$ [8], which rises significantly in the case of hemodynamic instability and left ventricular failure as was the case in our patient in whom another surgical redo AVR would pose an even greater risk as it would be his fifth surgical procedure. Redo
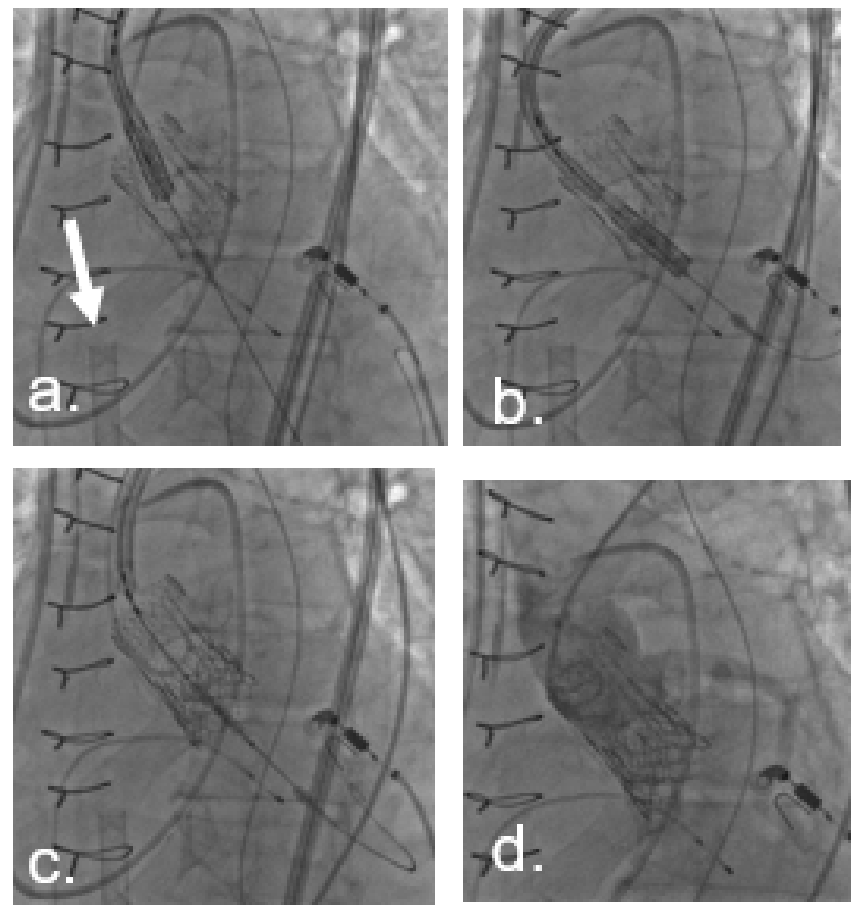

Figure 3. Step by step TAVR valve in valve. a. Edwards Sapien 3 valve $26 \mathrm{~mm}$. ECMO cannula (arrow). b. Positioning of TAVR device. c. Rapid pacing $(180 / \mathrm{min})$ and implantation of Sapien $326 \mathrm{~mm}$ valve. d. Final valve position with valve upper frame below LM. No regurgitation on aortic angiography. LM and RCA: no compromise of coronary flow 

shock

surgery is also associated with a prolonged recovery and increased morbidity. As valve in valve TAVR has recently proved feasible [9] and with no different early mortality rates compared to those for surgery in spite of higher preprocedural risk [4] it provides a reasonable and less invasive option in such patients. Recent data also confirm that TAVR is increasingly becoming not only an elective procedure but that nearly $10 \%$ of contemporary TAVRs are urgent or emergent and nearly $8 \%$ are performed in patients with LV ejection fraction $<30 \%$ [10]. However, in patients presenting with concomitant cardiogenic shock, TAVR was associated with nearly 33\% 30-day and 60\% 1-year mortality [11]. Predictors of 30-day mortality in these patients were baseline cardiac output $<3.01 / \mathrm{min}$, reduced cardiac power index, impaired renal function, mechanical ventilation as well as severe acute kidney injury after TAVR [11]. Pre- and postprocedural hemodynamic stabilization is therefore crucial for TAVR success in these patients.

VA ECMO proved to be a successful bridging therapy for hemodynamic stabilization in cardiogenic shock and there are reports of its use in high risk TAVR patients [12,13] although risk stratification for ECMO use in TAVR patients is still not clear and it was according to a recent review so far used in only a small percentage (4\%) of TAVR patients and mostly as emergency rescue strategy in cases of TAVR complications [5]. The outcomes of two series of patients $[14,15]$ in whom ECMO was used as a prophylactic measure in selected highrisk patients were comparable to conventional TAVR patients whereas emergency ECMO in TAVR was associated with significantly lower procedural success and survival [16]. Prophylactic ECMO before TAVR therefore seems a very effective use of mechanical support resources but risk stratification of patients that would benefit from its preprocedural implementation apart from patients in clear cardiogenic shock where TAVR and ECMO both present a rescue strategy is still to be defined, especially as ECMO itself carries significant risk of complications.

Another aspect of hemodynamic stabilization attempt in our report is the use of combination of VA ECMO and IABP, which are two of most commonly used temporary bridging mechanical support devices. Although they represent both ends of cardiac unloading capacity and although IABP was lately somewhat disregarded as an effective mechanical support [17], reports emerge on better survival and VA ECMO weaning success when used in combination with IABP [6]. These observations are hypothesized to be the result of a somewhat different mechanisms of IABP and ECMO cardiac support with IABP reducing or neutralizing some of the unwanted effects of percutaneous VA ECMO such as the afterload increase and by increasing coronary perfusion during ECMO support [18]. We believe such strategy is not applicable only to cardiogenic shock patients requiring VA ECMO but also to ECMO supported TAVR patients with severely reduced EF, where IABP could reduce additional afterload increase during hours of ECMO support and could enable an even more gradual and safe weaning of mechanical unloading of the stunned myocardium after ECMO removal.

\section{Conclusion}

We conclude that prophylactic percutaneous VA ECMO is an optimal hemodynamic stabilization method for rescue TAVR in cardiogenic shock and it also seems an attractive option for better results in high risk TAVR patients that still need to be stratified according to risk-benefit ratio. Combination of VA ECMO and IABP use might have a place in these patients as well to allow for a smooth myocardial recovery although this strategy still needs to be evaluated and proved in general.

\section{Disclosure}

The authors declare that they have no competing interests.

\section{References}

1. Thielmann M, Wendt D, Eggebrecht H, Kahlert P, Massoudy P, et al. (2009) Transcatheter aortic valve implantation in patients with very high risk for conventional aortic valve replacement. Ann Thorac Surg 88: 1468-1474. [Crossref]

2. Leon MB, Smith CR, Mack MJ, Makkar RR, Svensson LG, et al. (2016) Transcatheter or surgical aortic-valve replacement in intermediate-risk patients. $N$ Engl J Med 374 : 1609-1620. [Crossref]

3. Kogoj P, Furlan T, Bunc M (2014) Balloon aortic valvuloplasty for severe aortic stenosis: acute and long-term outcomes. Exp Clin Cardiol 20: 1961-1969.

4. Silaschi M, Wendler O, Seiffert M, Castro L, Lubos E, et al. (2017) Transcatheter valvein-valve implantation versus redo surgical aortic valve replacement in patients with failed aortic bioprostheses. Interact Cardiovasc Thorac Surg 24: 63-70. [Crossref]

5. Vallabhajosyula S, Patlolla SH, Sandhyavenu H, Vallabhajosyula S, Barsness GW, et al. (2018) Periprocedural cardiopulmonary bypass or venoarterial extracorporeal membrane oxygenation during transcatheter aortic valve replacement: A systematic review. J Am Heart Assoc 7: e009608. [Crossref]

6. Aso S, Matsui H, Fushimi K, Yasunaga H (2016) The effect of intraaortic balloon pumping under venoarterial extracorporeal membrane oxygenation on mortality of cardiogenic patients: an analysis using a nationwide inpatient database. Crit Care Med 44: 1974-1979. [Crossref]

7. Goslar T, Knafelj R, Radsel P, Fister M, Golicnik A, et al. (2016) Emergency percutaneous implantation of veno-arterial extracorporeal membrane oxygenation in the catheterisation laboratory. EuroIntervention 12: 1465-1472.

8. Chan V, Malas T, Lapierre H, Boodhwani M, Lam BK, et al. (2011) Reoperation of left heart valve bioprostheses according to age at implantation. Circulation 124: S75-80.

9. Webb JG, Dvir D (2013) Transcatheter aortic valve replacement for bioprosthetic aortic valve failure: the valve-in-valve procedure. Circulation 127: 2542-2550. [Crossref]

10. Holmes DR Jr, Nishimura RA, Grover FL, Brindis RG, Carroll JD, et al. (2016) Annual outcomes with transcatheter valve therapy: from the STS/ACC TVT registry. Ann Thorac Surg 101: 789-800.

11. Frerker C, Schewel J, Schluter M, Schewel D, Ramadan H, et al. (2016) Emergency transcatheter aortic valve replacement in patients with cardiogenic shock due to acutely decompensated aortic stenosis. EuroIntervention 11: 1530-1536.

12. Gopalamurugan AB, Meenakshi MS, Mohamed AR (2017) India's first successful ECMO assisted TAVI: a strategy for high risk TAVI patients. IHJ Cardiovascular Case Reports 2017: 4-6.

13. Pergolini A, Zampi G, Tinti MD, Polizzi V, Pino PG, et al. (2016) Combined venoarterial extracorporeal membrane oxygenation and transcatheter aortic valve implantation for the treatment of acute aortic prosthesis dysfunction in a high-risk patient. Rev Port Cardiol 35: 445.e1-445.e4

14. Husser O, Holzamer A, Philipp A, Nunez J, Bodi V, et al. (2013) Emergency and prophylactic use of miniaturized veno-arterial extracorporeal membrane oxygenation in transcatheter aortic valve implantation. Catheter Cardiovasc Interv 82: E542-E551.

15. Seco M, Forrest P, Jackson SA, Martinez G, Andvik S, et al. (2014) Extracorporeal membrane oxygenation for very high-risk transcatheter aortic valve implantation. Heart Lung Circ 23: 957-962.

16. Makdisi G, Makdisi PB, Wang IW (2016) Use of extracorporeal membranous oxygenator in transcatheter aortic valve replacement. Ann Transl Med 4: 306. [Crossref]

17. Unverzagt S, Buerke M, de Waha A, Haerting J, Pietzner D, et al. (2015) Intra-aortic balloon pump counterpulsation (IABP) for myocardial infarction complicated by cardiogenic shock. Cochrane Database of Systematic Reviews 7: CD007398.

18. Nuding S, Werdan K (2017) IABP plus ECMO-Is one and one more than two? J Thorac Dis 9: 961-964. [Crossref]

Copyright: (C)2019 Golicnik A. This is an open-access article distributed under the terms of the Creative Commons Attribution License, which permits unrestricted use, distribution, and reproduction in any medium, provided the original author and source are credited. 